\title{
Multigene Testing for Hereditary Cancer: When, Why, and How
}

\author{
Presented by Kenneth Offit, MD, MPH
}

\begin{abstract}
Multigene testing is a complicated area, with advantages and disadvantages of testing for hereditary cancer syndromes. Currently, NCCN does not endorse routing multiplex testing outside of a research setting, and/or intensive genetic counseling regarding risks and benefits. The 2017 NCCN Clinical Practice Guidelines in Oncology for Genetic/Familial High-Risk Assessment: Breast and Ovarian and Colorectal provide suggestions for mutation carriers identified by panel tests.
\end{abstract}

J Natl Compr Canc Netw 2017;15(5.5):741-743

Multigene (ie, multipanel) testing is a complicated area. At the NCCN 22nd Annual Conference, Kenneth Offit, MD, MPH, Chief, Clinical Genetics Services, Memorial Sloan Kettering Cancer Center (MSKCC), reviewed the advantages and disadvantages of multigene testing for hereditary cancer syndromes and discussed situations in which multigene testing should be considered.

"Someday, we'll be able to spit into our smart phones, and get a genetic readout and online counseling, but we are not there yet," Dr. Offit stated at the outset of his talk.

Currently, NCCN does not endorse routing multiplex testing outside of a research setting, and/or intensive genetic counseling regarding risks and benefits. The 2017 NCCN Clinical Practice Guidelines in Oncology (NCCN Guidelines) for Genetic/Familial High-Risk Assessment: Breast and Ovarian ${ }^{1}$ and Colorectal ${ }^{2}$ provide suggestions for mutation carriers identified by panel tests.

\section{Hereditary Cancers}

Current estimates are that the hereditary burden of cancer is around $16 \%$, and common cancer susceptibilities include, for example, Lynch syndrome, BRCA1/2, he-

Presented by Kenneth Offit, MD, MPH, Clinical Genetics Service, Memorial Sloan Kettering Cancer Center, New York, New York.

Dr. Offit has disclosed that he has no financial interests, arrangements, affiliations, or commercial interests with the manufacturers of any products discussed in this article or their competitors.

Correspondence: Kenneth Offit, MD, MPH, Memorial Sloan Kettering Cancer Center, 1275 York Avenue, New York, NY 10065.

E-mail: offitk@mskcc.org reditary breast and ovarian cancer syndrome, and lobular breast cancer with $\mathrm{CDH} 1$ mutations (associated with hereditary diffuse gastric cancer). Dr. Offit discussed hereditary cancers and suggested interventions (Table 1 ).

"The recent interest has been in genes associated with DNA repair pathways for cancers of the breast, ovary, pancreas, prostate, and pancreas. For instance, $20 \%$ of ovarian cancers will have germline mutations in DNA repair genes, both mismatch and homologous repair genes, which can be identified with multigene panel testing. Strikingly, $>40 \%$ of ovarian cancer cases in those of Jewish ancestry will have inherited cancer susceptibility mutations that can be screened," he said.

In men with metastatic prostate cancer, Dr. Offit cited a study that showed that $11.8 \%$ will have mutations in germline DNA repair genes compared with $4.6 \%$ with localized prostate cancer. ${ }^{3}$ DNA repair gene testing could soon become a standard of care for men with advanced prostate cancer, Dr. Offit emphasized. This testing is now being offered to men with advanced prostate cancer at MSKCC as part of a research study.

Single nucleotide polymorphisms (SNPs), associated with hundreds of genes, have been identified in studies involving hundreds of thousands of cancer cases and controls. In the future, and currently being performed in research studies in breast and prostate cancers, SNPs will be grouped together to estimate polygenic risks. Dr. Offit predicted that these will be used in clinical practice as part of polygenic models incorporating the rarer high-risk genes several years down the road. 
Offit

\begin{tabular}{|c|c|}
\hline Tumor Site (Gene) & Intervention (Surgery to be discussed; Drug) \\
\hline GIST tumor $(K I T)$ & Screening; Imatinib as therapy \\
\hline Thyroid (RET) & Thyroidectomy; vandetanib and cabozantinib as therapy \\
\hline Stomach $(C D H 1)$ & Gastrectomy \\
\hline Kidney (STK11, VHL, BHD, TS) & Screening; everolimus (mTOR) as therapy \\
\hline Basal cell $(P T C H)$ & Screening; vismodegib (hedgehog) as therapy \\
\hline Lynch syndrome $(M L H 1, M S H 2 \ldots)$ & Screening; colectomy, hysterectomy; PD-1 blockade as therapy, aspirin for colon cancer prevention \\
\hline Familial polyposis $(A P C)$ & Screening; colectomy, proctocolectomy; clinical trials for chemoprevention \\
\hline $\begin{array}{l}\text { Hereditary breast cancer } \\
(B R C A 1 / 2, \text { PALB2, CHEK } 2 \ldots)\end{array}$ & $\begin{array}{l}\text { Screening; mastectomy; salpingo-oophorectomy (PARPi as fourth-line treatment in patients with BRCA- } \\
\text { positive ovarian cancer; trials in progress for other cancers) }\end{array}$ \\
\hline
\end{tabular}

apinion of author, not NCCN.

\section{Multigene Testing}

Several factors have recently converged to encourage the commercialization of multigene testing, including the publicity surrounding Angelina Jolie's revelation about being a BRCA1 carrier and undergoing a double mastectomy, the Supreme Court ruling against BRCA1/2 as a proprietary test, currently available sequencing technology, and the availability of the internet.

Although multigene testing is now commercially widely available, the interpretation of this testing is challenging because in many cases clinical actionability is not clear. According to one study, approximately $20 \%$ to $30 \%$ of genes identified by these panels are variants of uncertain significance (VUS). ${ }^{4}$ Where results are more definitive, these results may pose risks to patients, such as for those with p53 mutations, where there is emerging evidence for effectiveness of interventions, and for those with $\mathrm{CDH} 1$ mutations, which require discussion of preventive gastrectomy.

Dr. Offit encouraged clinicians to have patients who have undergone multiplex testing enroll online in the PROMPT (Prospective Registry of MultiPlex Testing) registry, a joint initiative by MSKCC, Dana-Farber Cancer Institute, Mayo Clinic Cancer Center, and Abramson Cancer Center at Penn Medicine. Gathering these data will be helpful for making future decisions about when to test.

"The companies all agree this needs to be done. If a patient has a variant and doesn't know what it means, you can encourage them to join this online database." He compared the technology to "online ancestry services," except that in the PROMPT registry the initial phase is anonymous while individuals benefit from the information of other individuals who may share their same genetic variant. Thus far, the PROMPT registry has identified a $26 \%$ discordance rate among laboratories and an $11 \%$ rate with conflicting interpretations, ranging from pathogenic/likely pathogenic to VUS, a discrepancy that has implications for altering medical management. "The field needs harmonization," he stated, stressing the importance of the federally funded ClinVar repository, and noting that some laboratories may be basing their interpretation on data sets not made publicly available.

Current data suggest that BRIP1 is an ovarian cancer risk gene, not a breast cancer risk gene, and this distinction is noted in the 2017 NCCN Guidelines. Other "moderate-risk" genes may or may not be associated with a risk for breast cancer, such as NBN, BARD1, and RAD51C/D. "The challenge is the actionability of these findings. For example, currently there is no evidence to remove ovaries for patients with PALB2 or BARD1 mutations and no family history [of ovarian cancer]," Dr. Offit commented, noting that, in the case of PALB2, he anticipated that future studies may strengthen the ovarian cancer association.

"Currently the American Cancer Society has suggested that lifetime risks be used to determine whether a patient should undergo MRI screening for breast cancer," Dr. Offit continued. "Lifetime risks are inherently problematic. Different definitions of 'lifetime' yield different outcomes. 'Remaining lifetime risk' is higher for younger patients."

According to Dr. Offit, risk-adapted interventions based on risk thresholds are a better alternative. Age-specific risk ratios can be used to calculate agespecific incidence and cancer lifetime threshold risk. Genes other than BRCA and SNPs should be in- 
cluded. Models should incorporate other risk factors (eg, breast density) along with genes that have been identified. Agreement is needed on thresholds for action other than lifetime risk. Cost-effectiveness studies of intervention should be guided by risk threshold approaches, Dr. Offit advised.

An example of a guideline based on risk threshold can be drawn from the Tamoxifen prevention trial that used a $1.67 \%$ 5-year risk of developing breast cancer for a 60-year-old woman enrolled in the trial. ${ }^{6}$ Population-based mammogram screening is generally accepted in the United States starting at age 45 years with a 5 -year risk of $0.94 \%$. Dr. Offit cited a recent study suggesting that carriers of mutations in moderate-penetrance genes should have MRI screening when their risk reaches the level at which populationbased breast cancer screening is recommended. ${ }^{\text {? }}$

\section{Updated NCCN Guidelines}

The 2017 NCCN Guidelines for Genetic/Familial High-Risk Assessment: Breast and Ovarian are greatly expanded from the 2016 version-there are now 6 pages listing a number of genes that can be identified by multiplex testing and suggestions for management when those genes are present. Some of the genes pose no increased risk for breast cancer, others pose no increased risk of ovarian cancer, and others put patients at increased risk for one or both cancers.

Genes included in the new recommendations are ATM, BRCA1, BRCA2, BRIP1, CDH1, CHEK2, NBN, NF1, PALB2, PTEN, RAD51C, RAD51D, STK1, and TP53; MSH2, MLH1, MSH6, PMS2, and EPCAM are grouped together and do not increase the risk for breast cancer, but are associated with increased risk of ovarian and colon cancer syndromes.
"[These NCCN Guidelines] are an excellent first approximation. These data are helpful, but we need to be very careful. This is a fast moving field. We need to adapt our screening as better estimates of risk thresholds emerge," Dr. Offit advised.

Reimbursement for multiplex testing is a barrier for clinical usefulness. According to a recent study in JNCCN ${ }^{8}$ insurers want a strong evidence base to justify gene panel testing. Although patients should always check directly, insurers often will not reimburse for this testing, according to posted coverage guidelines available online. Insurers appear to differ regarding their coverage of this testing; for example, Cigna requires clinicians to meet guidelines, UnitedHealthcare may or may not pay for testing, and Aetna generally does not cover multigene testing.

\section{For Now?}

During his discussion, Dr. Offit indicated that panel testing should be considered for any patient with a BRCA1/2 mutation and all patients with, or a family history of, ovarian cancer. Dr. Offit stated that for colon and renal cancers, panel testing may be shown to be more cost-effective then serial testing.

"'Agnostic' [DNA sequencing] will show findings that would not be evident with typical testing based on family history. In our research studies, we have seen tumor types we don't expect. For example, we have seen prostate cancer with Lynch mutations. This patient inherited an MSH2 mutation, leading to a risk for colon cancer in his relatives," he explained. The time may be approaching for agnostic genetic testing, Dr. Offit predicted, "but this will require new approaches to high-throughput genetic counseling to accompany recent advances in highthroughput DNA sequencing."

\section{References}

1. Daly MB, Pilarski R, Berry M, et al. NCCN Clinical Practice Guidelines in Oncology for Genetic/Familial High-Risk Assessment: Breast and Ovarian. Version 2.2017. Accessed April 5, 2017.

2. Provenzale D, Gupta S, Ahnen DJ, et al. NCCN Clinical Practice Guidelines in Oncology for Genetic/Familial High-Risk Assessment: Colorectal. Version 2.2016. Accessed April 5, 2017.

3. Pritchard CC, Mateo J, Walsh MF, et al. Inherited DNA-repair gene mutations in men with metastatic prostate cancer. $\mathrm{N}$ Engl J Med 2016;375:443-453.

4. Domchek SM, Bradbury A, Garber JE, et al. Multiplex genetic testing for cancer susceptibility. J Clin Oncol 2013;31:1267-1270.

5. Balmaña J, Digiovanni L, Gaddam P, et al. Conflicting interpretation of genetic variants and cancer risk by commercial laboratories as assessed by the prospective registry of multiplex testing. J Clin Oncol 2016;34:4071-4078.

6. Vogel VG. Reducing the risk of breast cancer with tamoxifen in women at increased risk. J Clin Oncol 2001;16(18 Suppl):87S-92S.

7. Tung N, Domchek S, M, Stadler Z, et al. Counseling framework for moderate-penetrance cancer-susceptibility mutations. Nat Rev Clin Oncol 2016;13:581-588.

8. Trosman JR, Weldon CB, Douglas MP, et al. Payer coverage for hereditary cancer panels: barriers, opportunities, and implications for the Precision Medicine Initiative. J Natl Compr Canc Netw 2017. 